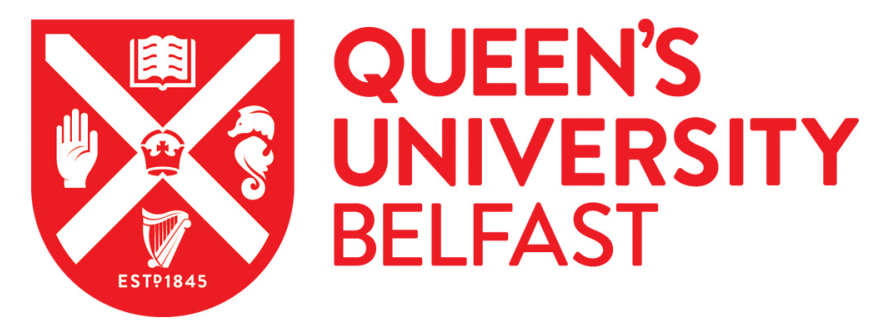

\title{
Active manipulation of the spatial energy distribution of laser- accelerated proton beams
}

Carroll, D. C., McKenna, P., Lundh, O., Lindau, F., Wahlstrom, C. G., Bandyopadhyay, S., Pepler, D., Neely, D., Kar, S., Simpson, P. T., Markey, K., Zepf, M., Bellei, C., Evans, R. G., Redaelli, R., Batani, D., Xu, M. H., \& Li, Y. T. (2007). Active manipulation of the spatial energy distribution of laser-accelerated proton beams. Physical Review E, 76(6), 065401. [065401]. https://doi.org/10.1103/PhysRevE.76.065401

Published in:

Physical Review E

Document Version:

Publisher's PDF, also known as Version of record

Queen's University Belfast - Research Portal:

Link to publication record in Queen's University Belfast Research Portal

\footnotetext{
General rights

Copyright for the publications made accessible via the Queen's University Belfast Research Portal is retained by the author(s) and / or other copyright owners and it is a condition of accessing these publications that users recognise and abide by the legal requirements associated with these rights.
}

Take down policy

The Research Portal is Queen's institutional repository that provides access to Queen's research output. Every effort has been made to ensure that content in the Research Portal does not infringe any person's rights, or applicable UK laws. If you discover content in the Research Portal that you believe breaches copyright or violates any law, please contact openaccess@qub.ac.uk. 


\title{
Active manipulation of the spatial energy distribution of laser-accelerated proton beams
}

\author{
D. C. Carroll and P. McKenna* \\ SUPA, Department of Physics, University of Strathclyde, Glasgow G4 ONG, United Kingdom
}

O. Lundh, F. Lindau, and C.-G. Wahlström

Department of Physics, Lund University, P.O. Box 118, S-22100 Lund, Sweden

S. Bandyopadhyay, D. Pepler, and D. Neely

STFC, Rutherford Appleton Laboratory, Didcot OX11 OQX, United Kingdom

S. Kar, P. T. Simpson, K. Markey, and M. Zepf

School of Mathematics and Physics, Queen's University Belfast, Belfast BT7 1NN, United Kingdom

C. Bellei and R. G. Evans

The Blackett Laboratory, Imperial College London, London, SW7 2AZ, United Kingdom

R. Redaelli and D. Batani

Dipartimento di Fisica, Università di Milano Bicocca, 20126 Milano, Italy

M. H. Xu and Y. T. Li

Beijing National Laboratory for Condensed Matter Physics, Institute of Physics, Chinese Academy of Sciences, Beijing 100080, China

(Received 9 July 2007; revised manuscript received 28 September 2007; published 12 December 2007)

The spatial energy distributions of beams of protons accelerated by ultrahigh intensity $\left(>10^{19} \mathrm{~W} / \mathrm{cm}^{2}\right)$ picosecond laser pulse interactions with thin foil targets are investigated. Using separate, low intensity $\left(<10^{13} \mathrm{~W} / \mathrm{cm}^{2}\right)$ nanosecond laser pulses, focused onto the front surface of the target foil prior to the arrival of the high intensity pulse, it is demonstrated that the proton beam profile can be actively manipulated. In particular, results obtained with an annular intensity distribution at the focus of the low intensity beam are presented, showing smooth proton beams with a sharp circular boundary at all energies, which represents a significant improvement in the beam quality compared to irradiation with the picosecond beam alone.

DOI: 10.1103/PhysRevE.76.065401

The use of ultraintense $\left(>10^{19} \mathrm{~W} / \mathrm{cm}^{2}\right)$ laser pulses to drive multi-MeV ion acceleration has generated a lot of international interest recently [1]. Many of the exciting possible applications of these ion beams would benefit from active control of the properties of the beam. It has recently been demonstrated experimentally that it is possible to optically change the direction of the proton beam $[2,3]$, and it has been shown theoretically that it should be possible to change the spectral distribution of the beam by use of a double laser pulse arrangement [4].

In this paper we present a study on the active manipulation of the spatial distribution of laser accelerated protons using separate low intensity laser pulses. In particular, we report conditions for which smooth beams with sharp circular boundaries at all energies are consistently produced, and show that the divergence properties of the ion beam can be actively manipulated. We show that with the addition of a second low intensity laser pulse, we obtain not only an improvement in the spatial quality of the beam, but also an increase in beam flux and energy.

The experiment is performed using multiple beams from the Vulcan laser at the Rutherford Appleton Laboratory. A

\footnotetext{
*Author to whom correspondence should be addressed. p.mckenna@phys.strath.ac.uk
}

PACS number(s): 52.38.Kd, 41.75.Jv, 52.50.Jm

chirped pulse amplified (CPA) pulse of 1 ps duration [full width at half maximum (FWHM)] and up to $90 \mathrm{~J}$ energy is used to drive the proton acceleration. The $1053 \mathrm{~nm}$ wavelength beam is focused using an off-axis $f / 3$ parabolic mirror. A plasma mirror [5] is positioned in the focusing beam to suppress the intensity of the amplified spontaneous emission to less than $10^{12} \mathrm{~W} / \mathrm{cm}^{2}$. The mirror is operated at $15^{\circ}$ angle of incidence, in a $P$-polarized geometry, and has a measured reflectivity of $55 \%$, giving a pulse energy on target of up to $50 \mathrm{~J}$. The beam is incident onto target at an angle of $5^{\circ}$ and the spot size at focus is $9 \mu \mathrm{m}$ (FWHM), resulting in a peak intensity of up to $4 \times 10^{19} \mathrm{~W} / \mathrm{cm}^{2}$. The targets are planar $\mathrm{Cu}$ foils of $5,12.5$, and $20 \mu \mathrm{m}$ thickness, and with a measured average peak-to-mean surface roughness of $\sim 0.7 \mu \mathrm{m}$. The arrangement is illustrated schematically in Fig. 1.

A low energy $(<5 \mathrm{~J}), 1053 \mathrm{~nm}$ wavelength pulse with a temporal profile approximating a flat-top distribution with a duration of $6 \mathrm{~ns}$ is used to drive ablation on the front surface of the target foil. The leading edge of the pulse arrives on target $3.5 \mathrm{~ns}$ prior to the arrival of the CPA pulse. The "ablation" pulse is focused using an $f / 10$ lens and is incident at an angle of $25^{\circ}$ onto target. A binary phase plate is positioned in the beam, in order to produce an annular distribution of laser light at focus, with a mean diameter of $460 \mu \mathrm{m}$ and a thickness of $\sim 35 \mu \mathrm{m}$, as shown in the inset of Fig. 1. The phase plate is a glass (borosilicate) substrate, on which a surface relief has been produced (using photolithography). 


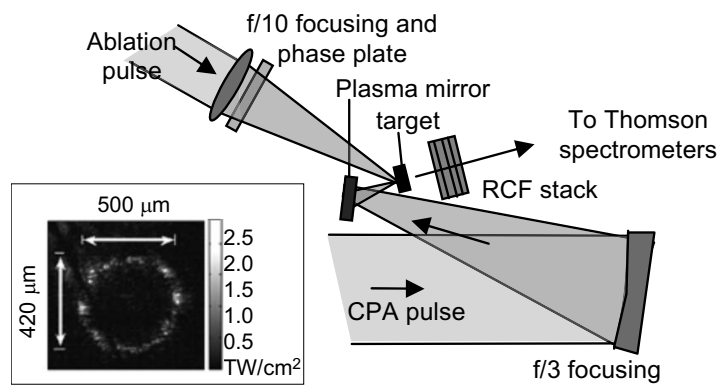

FIG. 1. Schematic diagram of the experiment. Proton acceleration is driven by a high intensity CPA pulse, which is focused using a f/3 off-axis parabolic mirror, with contrast enhancement provided by a plasma mirror positioned in the focusing beam. A binary phase plate introduced into the beam path of a separate low energy ("ablation") pulse, which is focused using an $f / 10$ lens, is used to produce a low intensity ring of laser light centered on the CPA focus. The measured spatial intensity distribution at the focus of the ablation pulse is shown in the inset. The proton spatial energy distribution is measured using a stack of RCF film and ion charge and spectral distributions are measured using Thomson ion spectrometers.

This induces a designed phase distribution in the laser beam transversing it, leading to the desired intensity distribution in the focus. The distribution of light produced around the ring varies in intensity between $3 \times 10^{11}$ and $3 \times 10^{12} \mathrm{~W} / \mathrm{cm}^{2}$.

The spatial distribution of the accelerated proton beam is measured simultaneously for different proton energies using a stack of radiochromic films (RCFs), which are preferentially sensitive to protons [6]. We confirm that the dose measured in the RCFs is predominately due to protons by introducing a periodical pattern in the intensity distribution of the proton beam using an absorbing mesh positioned behind the target on some shots. Each RCF in the stack measures the spatial distribution of the deposited dose for protons above an energy defined by the total thickness of the preceding stack layers. The stack is positioned $30 \mathrm{~mm}$ from the target and a slot machined in it provides a line of sight to an array of Thomson ion spectrometers.

With this arrangement, and in the absence of the ablation pulse, we detect the emission of proton beams with broad energy distributions from the rear of the targets. Using $5 \mu \mathrm{m}$-thick $\mathrm{Cu}$ targets, the energy distribution typically reaches up to $12 \mathrm{MeV}$, but when increasing the thickness to $20 \mu \mathrm{m}$, the maximum energy decreases to $9 \mathrm{MeV}$ and the beam divergence reduces. Representative examples of spatial distributions, obtained with 5 and $20 \mu \mathrm{m}$ targets, are shown in Figs. 2(a) and 2(c), respectively. Spatial modulations are typically observed in the proton dose, and may arise due to the roughness of the $\mathrm{Cu}$ target surface, modulations in the ultrahigh intensity drive laser beam, and/or instabilities in electron transport through the foil [7].

With the addition of the ablation beam, focused to produce the annular intensity distribution shown in the inset of Fig. 1, while using identical CPA beam and target parameters, we observe a significant improvement in the spatial distribution of the accelerated proton beam, together with increases in proton flux (typically about a factor of 3) and (a)

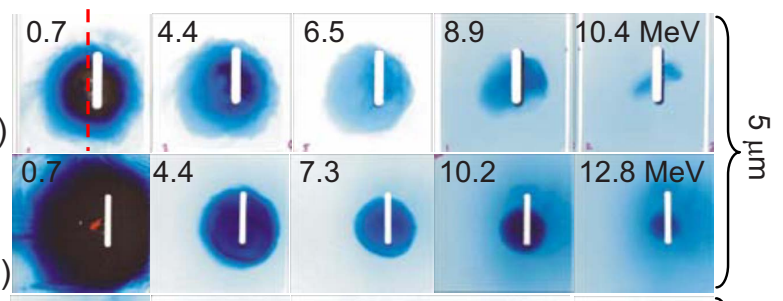

(c)

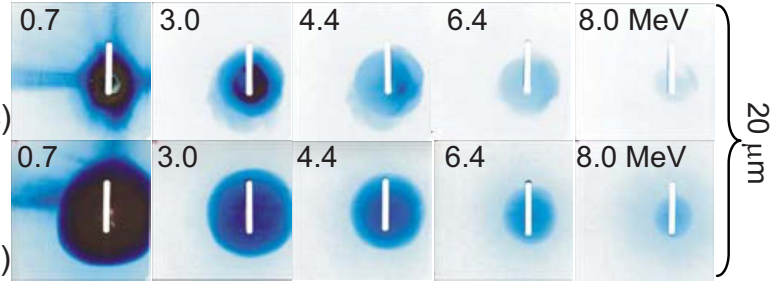

FIG. 2. (Color online) Representative examples of the measured proton spatial and energy distributions using RCF, obtained with a 5 - $\mu \mathrm{m}$ target (a) without and (b) with the "ablation ring" pulse; and the corresponding results for a $20-\mu \mathrm{m}$ target are shown in (c) and (d), respectively. The calculated intensity of the CPA pulse was between $2 \times 10^{19}$ and $3 \times 10^{19} \mathrm{~W} / \mathrm{cm}^{2}$ for all shots. These examples clearly show that the proton beam profile becomes more circular and more uniformly distributed in the presence of the low intensity ablation beam at both target thicknesses. The dashed line in (a) corresponds to the position of the lineout profiles sampled in Fig. 3, and applies to all RCFs in (a) and (b).

maximum proton energy. Corresponding measurements for the 5 and $20 \mu \mathrm{m}$ targets are shown in Figs. 2(b) and 2(d), respectively. Consistently we observe that with the ablation "ring" present, the profile of the proton beam becomes more like a "top-hat" distribution with a sharper circular boundary for most of the energy range. We quantify the improvement in the flatness of the beam by sampling the proton dose distribution in the region corresponding to $50 \%$ of the beam area, at the center of the beam, at each measured energy in the range 3.0-8.8 MeV. We find that the standard deviation of the proton dose in the film from a flat distribution varies from $54 \%$ of the mean value, at the lower energy, to $11 \%$ at the higher energy, when the target is irradiated by the CPA beam only. When the ablation pulse is added, the corresponding values are $16 \%$ and 4\%, respectively. Examples of beam profile lineouts as a function of energy, with and without the ablation pulse, are shown in Fig. 3.

Our findings can be interpreted in the framework of the
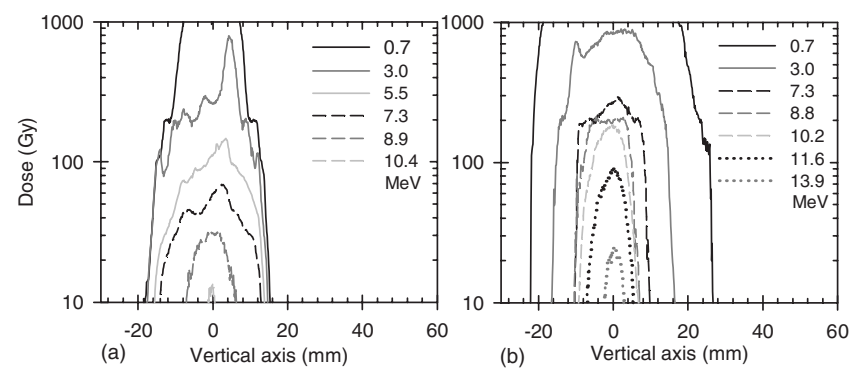

FIG. 3. Beam profile lineouts (along the position of the dashed line in Fig. 2) as a function of proton energy for the 5- $\mu \mathrm{m}$ target results shown in Fig. 2; (a) without and (b) with the ablation pulse. 

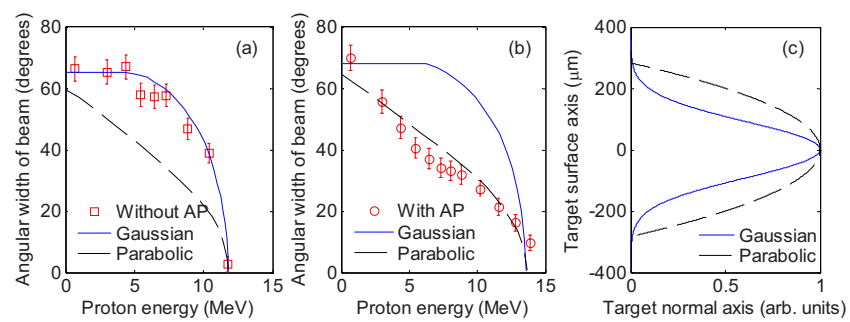

FIG. 4. (Color online) (a) Symbols correspond to the measured angular envelope of the proton beam as a function of proton energy for the 5- $\mu \mathrm{m}$ target sample measurements of Fig. 2, without the ablation pulse (AP); (b) corresponding measurements with the ablation pulse. The lines are the calculated divergence as a function of energy using the Gaussian (solid blue line) and the parabolic (dashed black line) sheath spatial distributions shown in (c). With the CPA beam only, a Gaussian distribution with a FWHM of $220 \mu \mathrm{m}$ reproduces the experimental results, as shown in (a), whereas a better fit to the experimental measurements in the presence of the long pulse ablation beam is obtained with a parabolic function with a FWHM of $400 \mu \mathrm{m}$, as shown in (b). The ratio of the peak electric fields used in the simulation results shown in (a) and (b) is 0.9 , as defined by the maximum measured proton energies.

target normal sheath acceleration (TNSA) mechanism [8]. Fast electrons, which are accelerated through the target by the CPA laser pulse, become electrostatically confined to the target rear surface, building a sheath with a quasi-static charge separation field, which ionizes and accelerates atoms at the rear surface. Protons are accelerated normal to isodensity contours in the sheath [6], and hence the measured spatial profile of the accelerated proton beam is sensitive to the shape of the sheath. The spatial profile of the sheath, and hence the ion front, is typically Gaussian shaped, as inferred from measurements by Fuchs et al. [6] and shown experimentally by Romagnani et al. [9]. Our observations of a change to the spatial profile of the proton beam can thus be interpreted as a change to the shape of the sheath, induced by the presence of the ablation beam.

Evidence of a change in the sheath shape is inferred from observed changes to the angular divergence as a function of energy. It has previously been shown, with single CPA beam irradiation of a target foil, that the angular envelope of the proton beam decreases with energy [10-12], and that this is due to a decrease in size of the emission region near the peak of the Gaussian-shaped electron sheath $[12,1]$. We find that the divergence characteristics of the proton beam changes significantly in the presence of the annular ablation beam, as shown in the example measurements of Figs. 2-4. The representative measurements in Fig. 4 illustrate the changes to the full angular envelope of the proton beam as a function of energy induced by the addition of the ablation beam. In the absence of the ablation ring, we consistently observe that the spatial extent of the beam is fairly constant for low proton energies and decreases only at the highest energies. This manifests itself in the plateau-like distribution at low energies in Fig. 4(a) and is quite different to the near-linear dependence of the divergence on proton energy consistently observed with the ablation ring present, Fig. 4(b), for otherwise identical target and laser conditions.
The measured changes to the proton beam divergence as a function of energy can be explained by changes to the spatial distribution of the sheath. To illustrate this, we devise a model to determine the sheath shape by fitting to the experimental measurements. The model calculates the spatial distribution of the expanding ion front, and the divergence and energy of protons produced along the ion front, as a function of time for a defined spatial and temporal distribution of the electric field. The temporal profile of the electric field is assumed to be Gaussian with a FWHM equal to $1.2 \mathrm{ps}$ - similar to the laser pulse duration. The peak strength of the field is chosen to reproduce the maximum detected energy of the accelerated protons. The model uses the threshold field for the ionization of hydrogen (assuming field ionization to be the dominant ionization mechanism at the rear surface of the target) to define the spatial extent of the proton source as a function of time. The ion front expands into vacuum and the velocity components and hence proton energy as a function of radius from the center of the sheath, at each temporal step ( 0.1 ps steps over a 4 ps range defined by the temporal profile of the electric field), is determined. The local normal at each point along the ion front surface is calculated as it evolves spatially and temporally, to determine the angle of proton emission.

Based on the measured sheath distributions reported by Romagnani et al. [8], for foil irradiation with a single CPA beam, we begin by assuming that the sheath shape has the form of a Gaussian distribution. We find a good fit to the experimental measurements, as shown in Fig. 4(a), when the FWHM of the distribution is equal to $220 \mu \mathrm{m}$. The spatial distribution of the sheath is shown in Fig. 4(c). Next, we consider what kind of change to the sheath shape would give rise to the measured changes in the divergence as a function of proton energy, when the ablation pulse is added. We find very good agreement with the experimental data, as shown in Fig. 4(b), when an inverse parabolic sheath profile with a FWHM of $400 \mu \mathrm{m}$ is used [Fig. 4(c)]. We note that an inverse parabolic profile of the ion front is used by Brambrink et al. [11] in their modeling of ion divergence from laserirradiated foil targets. With the ablation beam present, a slightly higher maximum electric field strength is required to produce the higher proton energies observed, and hence, for comparison, the calculated divergence as a function of energy for both sheath distributions and both peak electric fields are shown in Figs. 4(a) and 4(b).

The shape and lateral extent of the sheath, and the uniformity and spatial profile of the proton beam, are determined by a number of properties, including the number and distribution of hot electrons generated at the target front surface and accelerated into the foil, and the transport, including refluxing [13] of the hot electrons within the foil. Our findings that, with the ablation beam present, the ion front distribution and the profile of the proton beam changes, suggests that the properties of the hot electron generation at the front surface and/or the lateral spreading of the hot electrons within the foil are modified. The fact that the proton flux and maximum proton energy are consistently observed to increase in the presence of the ablation pulse suggests an increase in absorption of laser energy in the preformed plasma. In addition, in a separate investigation, using a similar ex- 
perimental arrangement and beam parameters, measurements using an interferometric laser probe diagnostic, suggest that the CPA laser beam filaments in the density gradients at the target front surface produced by the ablation pulse. The laser beam filamentation is observed over a larger area than the CPA focus and will produce multiple electron sources. This change in the fast electron generation could explain the observed changes to the sheath size and distribution, and may also produce smoothing of any irregularities in the sheath distribution that may otherwise give rise to structure in the spatial profile of the beam of accelerated protons. We note that although we start with an annular profile of the preformed plasma expansion (the initial ring diameter is approximately $460 \mu \mathrm{m}$ ) lateral expansion means that the inner diameter of the ring of ablated plasma is considerably smaller when the CPA pulse arrives on target. The influence of the shape and scale of the ablated plasma on electron acceleration at the target front surface will be the subject of further investigation.

We note that the observed changes to the proton beam are unlikely to result from rear surface effects induced by the breakout of an ablation-driven shock wave [2], as the same changes are observed for a range of $\mathrm{Cu}$ target thickness (5, 12.5 , and $20 \mu \mathrm{m})$. We also conclude that the observations are unlikely to result from a confinement of the lateral expansion of the electron cloud [14] within the target due to a local increase in the target resistivity in the material heated by the ablation pulse. Simulations with the LSP [15] and PETRA [16] codes indicate that this effect is not sufficient to explain the observations.

The findings reported in this paper indicate that it is possible to have active control on the spatial distribution of beams of laser-accelerated ions. Furthermore, this is achieved optically using a second low intensity laser pulse, and can therefore be deployed at high repetition rates. This has direct implication on possible applications of laseraccelerated ions. The change in beam profile to a more flattop distribution is useful, for example, for the application of laser-accelerated proton beams for imaging and radiography $[1,9]$.

We acknowledge the expertise of the Vulcan laser staff. O.L., F.L., and R.R. acknowledge financial support from the COST P-14 action.
[1] M. Borghesi, J. Fuchs, S. V. Bulanov, A. J. Mackinnon, P. K. Patel, and M. Roth, Fusion Sci. Technol. 49, 412 (2006).

[2] F. Lindau, O. Lundh, A. Persson, P. McKenna, K. Osvay, D. Batani, and C. G. Wahlström, Phys. Rev. Lett. 95, 175002 (2005).

[3] O. Lundh, F. Lindau, A. Persson, C. G. Wahlström, P. McKenna, and D. Batani, Phys. Rev. E 76, 026404 (2007).

[4] A. P. L. Robinson, D. Neely, P. McKenna, and R. G. Evans, Plasma Phys. Controlled Fusion 49, 373 (2007).

[5] B. Dromey, S. Kar, M. Zepf, and P. S. Foster, Rev. Sci. Instrum. 75, 645 (2004).

[6] J. Fuchs et al., Phys. Rev. Lett. 91, 255002 (2003).

[7] M. Roth et al., Phys. Rev. ST Accel. Beams 5, 061301 (2002).

[8] S. C. Wilks et al., Phys. Plasmas 8, 542 (2001).
[9] L. Romagnani et al., Phys. Rev. Lett. 95, 195001 (2005).

[10] R. A. Snavely et al., Phys. Rev. Lett. 85, 2945 (2000).

[11] E. Brambrink, J. Schreiber, T. Schlegel, P. Audebert, J. Cobble, J. Fuchs, B. M. Megelich, and M. Roth, Phys. Rev. Lett. 96, 154801 (2006).

[12] T. Cowan et al., Phys. Rev. Lett. 92, 204801 (2004).

[13] Y. Sentoku, T. E. Cowan, A. Kemp, and H. Ruhl, Phys. Plasmas 10, 2009 (2003).

[14] P. McKenna et al., Phys. Rev. Lett. 98, 145001 (2007).

[15] D. R. Welch, D. V. Rose, B. V. Oliver, and R. E. Clark, Nucl. Instrum. Methods Phys. Res. A 464, 134 (2001).

[16] J. Honrubia, A. Antonicci, and D. Moreno, Laser Part. Beams 22, 129 (2004). 